\title{
Mobility and Network Management in Virtualized Networks
}

\author{
Kostas Pentikousis • Ramon Agüero - Susana Sargento • \\ Rui L. Aguiar
}

Published online: 1 July 2012

(C) Springer Science+Business Media, LLC 2012

\section{Introduction}

Management, be it multi-access, resource, mobility or network management, is a key aspect in the design, deployment and operation of current and future networks. The challenges that mobile network management faces today are increasingly associated with the multiplicity of operating environments. For instance, heterogeneous resource sharing, dealing with a larger variety of user profiles, and operating diverse network infrastructures have become commonplace. In this context, it is natural that we witness proliferation and adoption of virtualization techniques in different areas of information and communication technologies (ICT), which then introduce their own management challenges.

Although certain virtualization techniques are already embraced in telecommunication networks, such as network sharing, the full potential that can be achieved from a completely virtualized network environment is yet to be

\author{
K. Pentikousis $(\bowtie)$ \\ Huawei Technologies, \\ Carnotstrasse 4, \\ 10587 Berlin, Germany \\ e-mail: k.pentikousis@huawei.com \\ R. Agüero \\ University of Cantabria, \\ Edificio Laboratorios I+D+I Telecomunicaciones, \\ Plaza de la Ciencia s/n, \\ 39005 Santander, Spain \\ e-mail: ramon@tlmat.unican.es \\ S. Sargento $\cdot$ R. L. Aguiar \\ University of Aveiro, Instituto de Telecomunicações, \\ Campus Universitário de Santiago, \\ 3810-193 Aveiro, Portugal \\ S. Sargento \\ e-mail: susana@ua.pt \\ R. L. Aguiar \\ e-mail: ruilaa@ua.pt
}

realized. In practice, we are not able to fully control these environments. Proponents of network virtualization point to the dramatically increased flexibility in running networks, enabling multiple networks to operate simultaneously over the same infrastructure, while supporting different technologies, protocols, topologies, and quality of service (QoS) and experience (QoE) requirements. All these are not possible with today's technology. Although virtualization has been studied extensively in other domains, its application to mobile networks is an emergent topic of active research and its impact on resource, mobility, and network management, as well as the related security and autonomicity challenges are open for investigation. What is certain though is that the interest in mobile networks and virtualization is geared to increase.

Before we go ahead with the core topic of this special issue, let us stand back for a moment and consider how mobile infrastructure networks have evolved. Traditionally, mobile operators deployed their own, dedicated, access network-specific infrastructure in order to offer different network services to their subscribers. This is not always the case anymore. As we move towards the widespread deployment of mobile broadband based on the fourth generation $(4 \mathrm{G})$ of cellular networks, and in parallel with the continuous expansion of previous generation networks, we see that key stakeholders (from operators to regulators) consider changing their modus operandi with respect to network infrastructure build out. We expect that increased infrastructure sharing will be very common in the mid-term. This should not be surprising. The way we understand and interact with communication commodities is continuously evolving. What is new, though, is that the pace of changes in this line of evolution has dramatically accelerated during the last decade, leading to a somewhat hard(er) to forecast path in terms of technology evolution. Although this spans throughout ICT technologies, protocols and infrastructures, wireless and mobile networks are perhaps the most representative example of such an evolution. 
Currently, the use of multi-access smartphones and tablets running several network services simultaneously has become very popular across the globe and one of the consequences is the rapid growth on the demands and requirements (both quantitatively and qualitatively) from end-users. Although the subjacent technologies are continuously improving to accommodate the increased demand (the faster rollout of High Speed Packet Access [HSPA] and Long Term Evolution [LTE] when compared to $3 \mathrm{G}$ deployments are examples of such an evolution), there are still several gaps which need to be addressed. In parallel to the advances on the subjacent technologies and their capacity, the consequences brought about by the upcoming Future Internet network architecture proposals are poised to lead to changes in the current communications paradigm [1]. A paradigm shift will affect the way networks and services (particularly within mobile environments) are designed, dimensioned, deployed, and managed, especially when we consider that resources are to be jointly shared and managed.

Given this backdrop, virtualization techniques have attracted significant attention from the ICT research community as well as prominent industry players and operators. So far, virtualization has been widely applied to computing resources, such as storage and processing capacity, as well as to individual network elements, such as virtualized secure set identifiers (SSIDs) in IEEE 802.11 wireless LAN access points. In this special issue we look at the possibilities which are brought about by the use of advanced resource sharing techniques from communication resources to entire networks, including virtualization. This point of view opens a great number of possibilities (as well as introduces new challenges) for network operators, as to how to employ these techniques in order to offer better service to their users.

The papers included in this special issue address several topics, including:

- Mobile and multi-access network management, resource management and virtualization

- Multimedia applications and services for mobile/multiaccess networks

- Future Internet architectures for mobile network virtualization

- Virtualization in autonomic mobile networks

- IEEE and 3GPP management standards and enabling technologies

- Operator vs. user-centric mobile network management and virtualization

- New business models and economics of virtualized mobile networks

Furthermore, this special issue considers network virtualization in the context of other important developments in wireless communications and mobile networks. One such topic is the wider interest in capitalizing on wireless mesh networks as well as opportunistic networking. This type of topologies has recently drawn large attention, since they offer a great number of advantages, like the possibility to extend economically the coverage and capacity of infrastructure network deployments. Moreover, cognitive and automated management procedures are also addressed by papers of this issue. These mechanisms are crucial, since the increasing demand of services and users require more intelligence on the network side. On the other hand, operators need to increase their revenues, and are likely to include business-related aspects within the corresponding cognitive cycle.

Finally, further aspects that should also be addressed in the context of mobile network management relate to the so-called Internet-of-Things (IoT). The possibilities which have been opened thanks to the deployment of smart objects and their applications are very broad and such networks will undoubtedly play a key role in the near-term wireless communications evolution. This will be the topic of a future special issue in this Journal as well of special interest to MONAMI 2012.

\section{Mobile networks and virtualization}

This is the second of the series of MONET special issues focused on innovative architectures, mechanisms, protocols, and algorithms for mobile and wireless network management. The first special issue [2] addressed key topics in heterogeneous mobile networks, such as wireless access selection [3], autonomic management [4], multihoming management [5], and mobility management [6]. The papers included in this second special issue constitute the perfect complement to the Proceedings of the ICST Conference on Mobile Networks and Management (MONAMI) [7], which is organized for a fourth consecutive year in September 2012, in Hamburg, Germany.

After our open call for papers for this special issue, 24 submissions from 16 countries on 3 continents entered the rigorous peer-review process. One paper which was presented at MONAMI 2011, in Aveiro, Portugal, was significantly extended and, after entering the normal journal peerreview process, was accepted for publication. The remaining of this editorial serves as a short introduction to each of the five papers comprising this issue.

The first paper, entitled "Providing throughput and fairness guarantees in virtualized WLANs through Control Theory" by A. Banchs et al. delves into the issue of wireless infrastructure sharing. In particular, the authors explore virtualization of wireless access points (APs) based on IEEE 802.11 and use Control Theory to develop an algorithm that distributes network resources fairly among virtualized Wireless LAN APs. They evaluate their algorithm analytically and show that their proposal is able to fulfill a set of requirements, including the capacity to perform well under a 
variety of traffic conditions which may change at any point of time. In addition, the authors also discuss how the corresponding mechanisms and procedures can be applied to off-the-shelf WLAN access points.

R. Matos et al. in the paper entitled "Distributed approach to control and manage context-based multi-virtual networks" define a framework for wireless mesh networks that capitalizes on virtualization techniques and distributed control mechanisms. Their architectural goal is to enable the network to adjust the service level offered based on the end user context. The proposed framework uses context information to group users based on several criteria and offer, accordingly, the corresponding (personalized) network service. The authors advocate a distributed approach in wireless mesh network virtualization so as to share the network control responsibility burden. Finally, they compare their mechanisms with other design alternatives using analysis and simulation.

The third paper of this special issue, entitled "Scheme for expanding the capacity of wireless access infrastructures through the exploitation of opportunistic networks" is the result of article development through MONAMI 2011 [7]. A. Georgakopoulos et al. consider the use of opportunistic and mesh networks to extend both coverage and capacity of traditional infrastructure network deployments. The authors propose the adoption of dynamically created, opportunistic networks in order to offload saturated macro-cell base stations, improving performance in terms of energy consumption, error rates, and rejected calls. The authors evaluate their solution approach through simulation using two complementary tools.

One important aspect in mobile network management that so far has not been given extensive and rigorous treatment in the literature is resource management procedures that integrate tightly with the business goals of a mobile network operator. H. Galeana-Zapién et al. propose, in the fourth paper of this special issue, a cognitive framework which establishes low level (radio) resource mechanisms, based on business-level indicators. Their paper, entitled "A business-oriented management framework for mobile communication systems", puts forward a proposal generic enough so as to adapt to any technology, including virtualized infrastructures. The authors assess the validity of their methods using the OPNET simulation suite to examine performance for a UMTS cellular access network. The results presented point to noteworthy benefits compared to traditional approaches.

Last, but certainly not least, this special issue concludes with the work by T. Ojanperä et al. on multimedia service delivery over multi-access networks. Their paper, entitled "Integrating adaptive video streaming service with multiaccess network management", received the highest score from all open-call submissions after the thorough peerreview process was concluded. In effect, Ojanperä et al. won the unofficial best paper award for this special issue. The authors take the view that network heterogeneity is only to increase in the future. This trend will go hand in hand with the increased user demand for audio/visual content, and in particular video streaming services. Today, multi-access network management is far from automated. The authors expect that in the future, cognitive network management will play a key role in delivering end-user multimedia services. To this end, the paper introduces a network control architecture that employs cognitive mechanisms to optimize video streaming over multi-access networks. The architecture is based on a framework, including the corresponding control and signaling elements, which aims to minimize management effort for both end users and network operators. The authors assess the feasibility of their proposal using a prototype implementation and report experimental results, under a variety of access patterns, from a testbed that includes a scalable video coding (SVC) video streaming service.

We would like to thank all authors and expert reviewers who helped us introduce this timely topic. We close this editorial note by acknowledging the warm and continuous support of Prof. Imrich Chlamtac, the Journal's Editor-inChief, as well as Dr. Sara Fruner for her brilliant coordination of this special issue.

\section{References}

1. Pentikousis K (2010) In search of energy-efficient mobile networking. IEEE Commun Mag 48(1):95-103

2. Pentikousis $\mathrm{K}$ et al (2011) Mobility and network management in heterogeneous networks. Mobile Netw Appl J 16(4):409-411, Springer

3. Choque $\mathrm{J}$ et al (2011) Optimum selection of access networks within heterogeneous wireless environments based on Linear Programming techniques. Mobile Netw Appl J 16(4):412-423, Springer

4. Aristomenopoulos G et al (2011) An autonomic QoS-centric architecture for integrated heterogeneous wireless networks. Mobile Netw Appl J 16(4):490-504, Springer

5. Sousa B et al (2011) Multihoming management for future networks. Mobile Netw Appl J 16(4):505-517, Springer

6. Silva R et al (2011) Enabling heterogeneous mobility in Android devices. Mobile Netw Appl J 16(4):518-528, Springer

7. Pentikousis K, et al. Mobile Networks and Management, LNICST vol. 97, ISBN: 978-3-642-30421-7. Springer

Kostas Pentikousis is a senior research engineer with Huawei Technologies, Berlin, Germany since 2010. From 2005 to 2009 he was a senior research scientist with VTT Technical Research Centre of Finland. Kostas earned his bachelor's degree in informatics (1996) from Aristotle University of Thessaloniki, Greece, and his master's (2000) and doctoral degrees (2004) in computer science from the State University of New York at Stony Brook. Dr. Pentikousis has published in several areas, including network architecture and design, mobile networking and computing, transport protocols, applications and services, 
local and wide area networks, energy-efficient networking, and information-centric networking.

Ramón Agüero received a degree in Telecommunications Engineering from the University of Cantabria in 2001 and a PhD in 2008. He is currently an Associate Professor at the Communications Engineering Department at that university. He has participated in several collaborative research projects. His research focuses on WLAN and WPAN technologies, with special attention on performance analysis of TCP/IP protocols over them and multi-hop (mesh) networks. He is also interested in aspects concerning heterogeneous network deployments and the Wireless Sensor Networks area. He has published more than 100 technical papers in such areas.

Susana Sargento (www.av.it.pt/ssargento) received her PhD in 2003 in Electrical Engineering. She joined the Department of Computer Science of the University of Porto in September 2002, and is in the University of Aveiro and the Institute of Telecommunications since February 2004. She has been TPC Chair and organizing several conferences, such as MONAMI'11, NGI'09, IEEE ISCC'07, NTMS'12 and IEEE FEDNET (with IEEE NOMS'12). She has been also in the
Technical Program Committee of several international conferences and workshops (more than 15 in 2011), such as ACM MobiCom 2009 Workshop CHANTS and IEEE Globecom (2010). She has also been a reviewer of numerous international conferences and journals. Her main research interests are in the areas of Next Generation and Future Networks, more specifically QoS, mobility, self-* and cognitive networks, with more than 200 papers in these areas.

Rui Aguiar received a Ph.D. degree in electrical engineering in 2001 from the University of Aveiro. He is currently a professor at the University of Aveiro and is leading a research group (ATNOG) at the Institute of Telecommunications, Aveiro, on next-generation network architectures and protocols. His current research interests are centered on the implementation of advanced wireless networks, systems, with special emphasis on mobility and Future Internet challenges. He has more than 300 published papers in those areas. He has served as technical and general chair of several conferences, such as ICNS '05, ICT'06, ISCC'07 and MONAMI 2011. He currently serves as Associate Editor of several journals and Steering Committee member of MONAMI. 